\title{
3-D REAL-TIME ACCURACY CONTROL OF AUTOMATED ROAD CONSTRUCTION MACHINES
}

\author{
Rauno Heikkilä, Mika Jaakkola \\ Oulu University, Research of Construction Technology \\ P.O.Box 4400 (Kasarmintie 4), FIN-90014 Oulu, Finland \\ rauno.heikkila@oulu.fi,.mika.jaakkola@,oulu.fi
}

\begin{abstract}
This research studied the possibilities for utilizing different a priori types of measuring algorithms in order to improve accuracy and reliability of automated machine control in road construction. Generally in measurement science different a priori types of algorithms have widely been used to estimate beforehand the propagation of randomly appearing errors. While the exact mathematical solutions for derivative measurement quantities, as well as the random errors of fundamental quantities are known, the random errors of derivative quantities can always be evaluated using stochastic mathematics. In principle, this type of stochastic model can give us the magnitude of the highest attainable accuracy in practical measurement situations. In our research, new stochastic models to determine the random errors of robot tachymeter in kinematic measurement situations were developed and introduced. The tested tachymeter was TRIMBLE ATS. The needed random errors for the test solution were determined based on the technical specifications obtained from Trimble. In addition, two different kinematic calibrations for the use of robot tachymeters for road construction automation were developed and executed.
\end{abstract}

Keywords: automation in construction, accuracy control, stochastic models, intelligence in automation

\section{INTRODUCTION}

In practical road construction the direct use of automated machine control systems for construction work sets great demands for maintaining adequate control accuracy of the utilized technologies. The economic consequences of deviations can be very significant. Until today the possibilities of these a priori types of algorithms have not been applied in the domain of road construction automation. Also, manufacturers of tachymeters have not presented the exact positioning accuracy of their products. For example, Trimble presents that the accuracy of Trimble ATS 600 in dynamic operation is as follows: maximum acceleration of target (radial acceleration) $10 \mathrm{grad} / \mathrm{s}$ and $29 \mathrm{deg} / \mathrm{s}^{2}$, maximum velocity of target (radial speed) $25 \mathrm{grad} / \mathrm{s}$ or 23 $\mathrm{deg} / \mathrm{s}$, and axial speed $6 \mathrm{~m} / \mathrm{s}$. In addition, Trimble presents that accuracy at constant speed of $1 \mathrm{~m} / \mathrm{s}$ at $300 \mathrm{~m}$ (standard deviation) is $\pm 2 \mathrm{~mm}$ $+14 \mathrm{ppm}$ in horizontal and vertical distances. The question is: What kind of accuracy can we obtain in other situations?

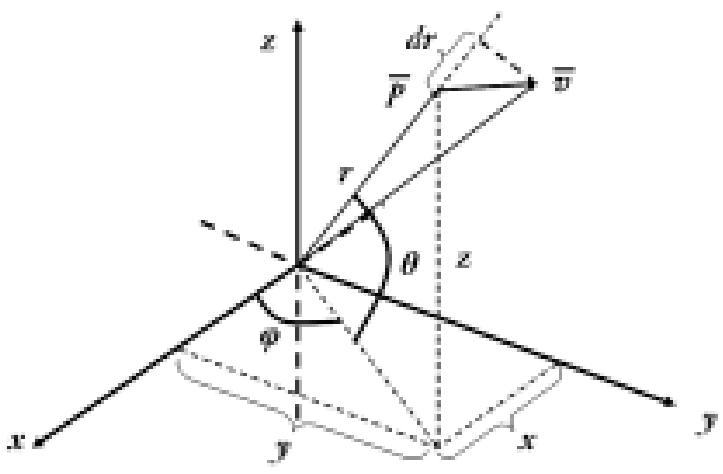

Figure 1. The dynamic situation of 3-D positioning measurement by a robot tachymeter in an arbitrary machine control application.

\section{STOCHASTIC MODELLING AND TESTING}

New stochastic models to determine the random errors of a robot tachymeter in a kinematic measurement situation were developed and introduced. The tested tachymeter was Trimble ATS. The needed random errors for the test solution were first determined based on the 
technical specifications obtained from Timble. In addition, three different kinematic calibrations for the use of robot tachymeters for road construction automation were developed and executed in conjunction with Helsinki University of Technology and the Finnish importer Geotrim Oy.

The required functional equations for cartesian $x$, $y$, and $z$ coordinates are

$\left\{\begin{array}{l}x=r \cos \theta \cos \varphi \\ y=r \cos \theta \sin \varphi \\ z=r \sin \theta\end{array}\right.$

where $r, \theta$, and $\varphi$ are the basic spherical coordinate observations of a tachymeter. To evaluate the uncertainty of the measurements, the propagation of random errors must be cleared. Assuming that normally distributed random variables are considered, the uncertainty of the independent spherical coordinates at a given point can be illustrated by the covariance matrix

$C_{p}=\left[\begin{array}{ccc}m_{r}{ }^{2} & 0 & 0 \\ 0 & m_{\theta}{ }^{2} & 0 \\ 0 & 0 & m_{\varphi}{ }^{2}\end{array}\right]$

where $m_{r^{2}}, m_{\theta^{2}}$, and $m_{\varphi^{2}}$ are the variances of spherical coordinates. The covariance matrix of cartesian coordinates is

$C_{c}=J_{c p} C_{p} J_{c p}{ }^{t}$

where

$C_{c}=\left[\begin{array}{lll}m_{x}{ }^{2} & m_{x y} & m_{x z} \\ m_{x y} & m_{y}{ }^{2} & m_{y z} \\ m_{x z} & m_{y z} & m_{z}{ }^{2}\end{array}\right]$

and

$$
J_{c p}=\left[\begin{array}{lll}
\frac{\partial x}{\partial r} & \frac{\partial x}{\partial \theta} & \frac{\partial x}{\partial \varphi} \\
\frac{\partial y}{\partial r} & \frac{\partial y}{\partial \theta} & \frac{\partial y}{\partial \varphi} \\
\frac{\partial z}{\partial r} & \frac{\partial z}{\partial \theta} & \frac{\partial z}{\partial \varphi}
\end{array}\right]
$$

The uncertainty of static measurement $C_{\text {static }}$ is mathematically fully determined since the uncertainties $m_{\mathrm{r}}, m_{\theta}$ and $m_{\varphi}$ are now known, Eq. (2). The needed variances and covariances can be solved from Eq. (1) and Eq. (3) and are

$$
\left\{\begin{array}{l}
m_{x^{2}}=(\cos \theta \cos \varphi)^{2} \cdot m_{r}{ }^{2}+(-r \sin \theta \cos \varphi)^{2} \cdot m_{\theta}{ }^{2}+ \\
(-r \cos \theta \sin \varphi)^{2} \cdot m_{\varphi}{ }^{2} \\
m_{y^{2}}=(\cos \theta \sin \varphi)^{2} \cdot m_{r}{ }^{2}+(-r \sin \theta \sin \varphi)^{2} \cdot m_{\theta}{ }^{2}+ \\
(r \cos \theta \cos \varphi)^{2} \cdot m_{\varphi}{ }^{2} \\
m_{z}{ }^{2}=(\sin \theta)^{2} \cdot m_{r}{ }^{2}+(r \cos \theta)^{2} \cdot m_{\theta}{ }^{2} \\
m_{x y}=\frac{1}{2} \sin 2 \varphi \cdot\left(\cos ^{2} \theta \cdot m_{r}{ }^{2}+r^{2} \sin ^{2} \theta \cdot m_{\theta}{ }^{2}\right. \\
\left.-r^{2} \cdot \cos ^{2} \theta \cdot m_{\varphi}{ }^{2}\right) \\
m_{x z}=\frac{1}{2} \sin 2 \theta \cdot\left(\cos \varphi \cdot m_{r}{ }^{2}-r^{2} \cdot \cos \varphi \cdot m_{\theta}{ }^{2}\right) \\
m_{y z}=\sin ^{2} \theta \cdot m_{r}{ }^{2}+r^{2} \cdot \cos ^{2} \theta \cdot m_{\theta}{ }^{2}
\end{array}\right.
$$$$
\text { 5) }
$$

In a dynamic measurement situation we should know the additional uncertainties of spherical observations $r, \theta$, and $\varphi$. When simplified, based on the Fig. 1, we can assume that additional uncertainties arise from the velocities and accelerations of spherical coordinates. With Eq. (3) we can deduce the uncertainties of the cartesian coordinates in the case of static measurement. This is based on the known uncertainties $m_{r}{ }^{2}, m_{\theta}{ }^{2}$, and $m_{\varphi}{ }^{2}$ of the fundamental quantities $r, \theta$, and $\varphi$. The uncertainty of a kinematic positioning situation can be estimated if we know the additional uncertainty caused for velocities and accelerations of $v_{r}, v_{\theta}, v_{\varphi}$ and $a_{r}, a_{\theta}$ and $a_{\varphi}$. Thus, the task is to calibrate the dependencies between the spherical velocities and accelerations, and the additional uncertainties.

One special case in a dynamic positioning situation is the shaking of a prism. Based on the practical observations in the case of a road grader, we can estimate the additional 
uncertainty being generated by shaking with the equation

$$
C_{\text {shaking }}=\left[\begin{array}{ccc}
m_{x}{ }^{2} & 0 & 0 \\
0 & m_{y}{ }^{2} & 0 \\
0 & 0 & m_{z}{ }^{2}
\end{array}\right]
$$

where we can further assume $m_{x}{ }^{2}=m_{y}{ }^{2}$ since the shaking in different horizontal directions is identical.

An Excel sheet application was developed to test and utilize these models. The first calculations were executed by using own estimates for the additional uncertainties caused by velocities, accelerations, and shaking. For a more accurate and usable utilization two different types of dynamic calibration tests were executed using Trimble tachymeters, Figs. 2-4. A third calibration with Leica tachymeters has also been planned.

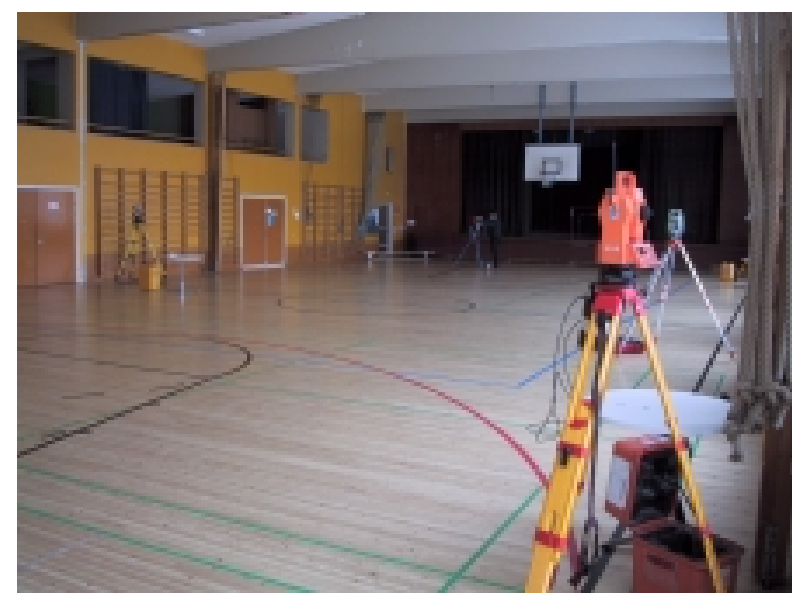

Figure 2. Calibration test using three Trimble tachymeters at the gym hall of Oulu International school.

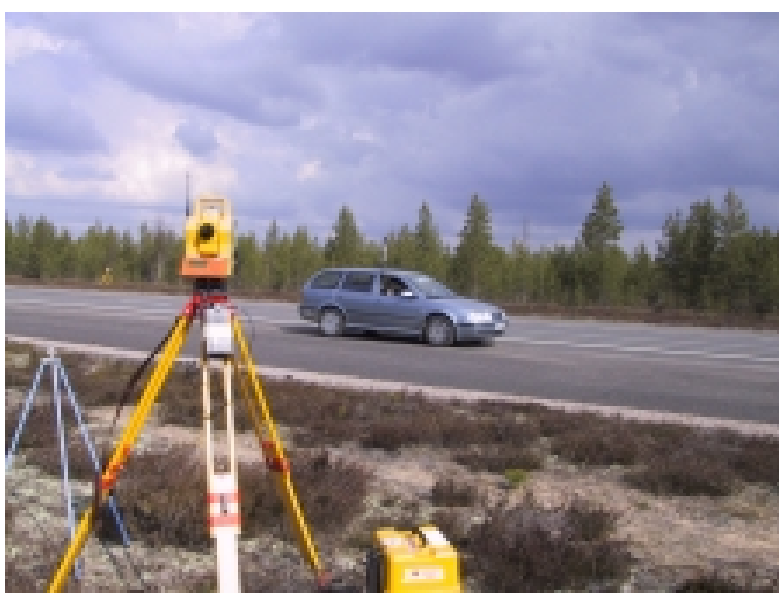

Figure 3. Calibration test using three Trimble tachymeters at the Kestilä Airfield

\section{TEST RESULTS}

The first test results of the models are presented in the Figures 4-6. In these example calculations $r=200 \mathrm{~m}, \varphi=30^{\circ}, \theta=25^{\circ}, v_{r}=2,4 \mathrm{~m} / \mathrm{s}, v_{\varphi}=0,006^{\circ} / \mathrm{s}$, $v_{\theta}=0,001 \% / \mathrm{s}, \quad m_{r}=5.0 \mathrm{~mm}, \quad m_{\varphi}=0,004 \%$, $m_{\theta}=0,004 \%, \mathrm{~m}_{\text {xy-shaking }}=100 \mathrm{~mm}, \mathrm{~m}_{\text {z-shaking }}=5$ $\mathrm{mm}, \quad m_{v r}=2,4 \quad m m, \quad m_{v \varphi}=0,0000024^{\circ}$, $m_{v \theta}=0,0000004^{\circ}$ and $m_{a r}=2,4 \mathrm{~mm}, m_{a \varphi}=0,003^{\circ}$, $m_{a \theta}=0,003^{\circ}$. These values are evaluated only for this calculation test.

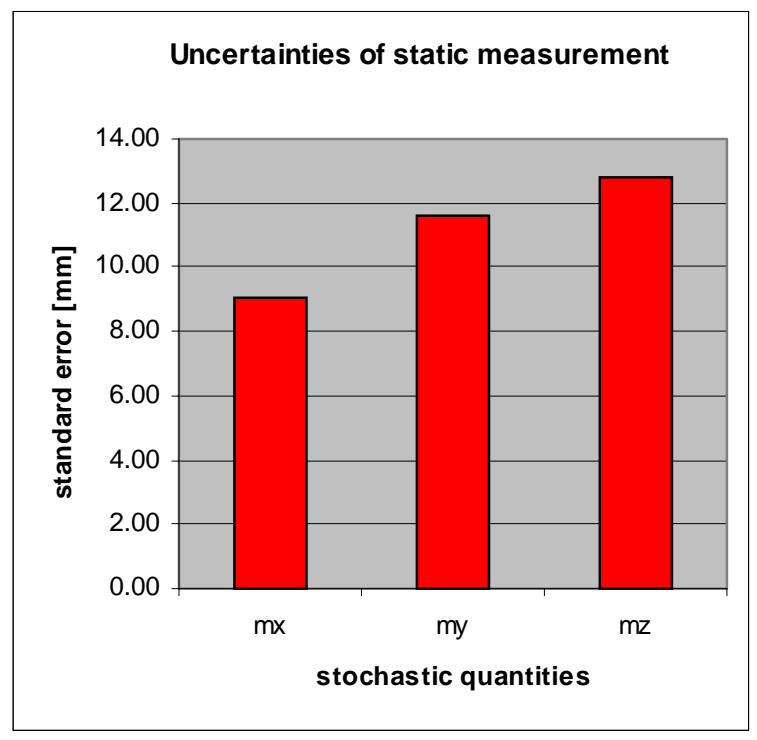

Figure 4. Random errors of static measurement in the test situation. 


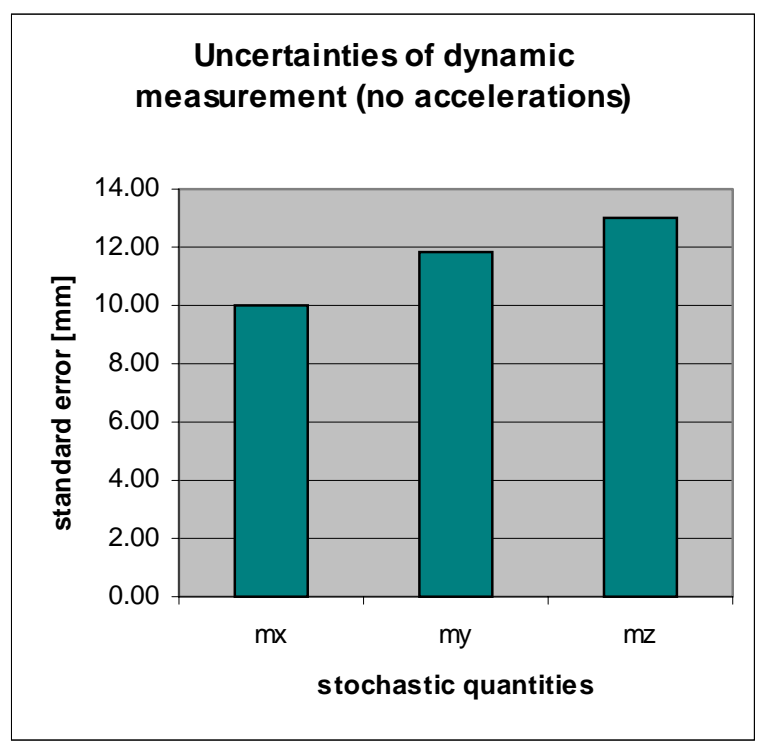

Figure 5. Random errors of dynamic measurement (static + shaking + velocities) in the test situation.

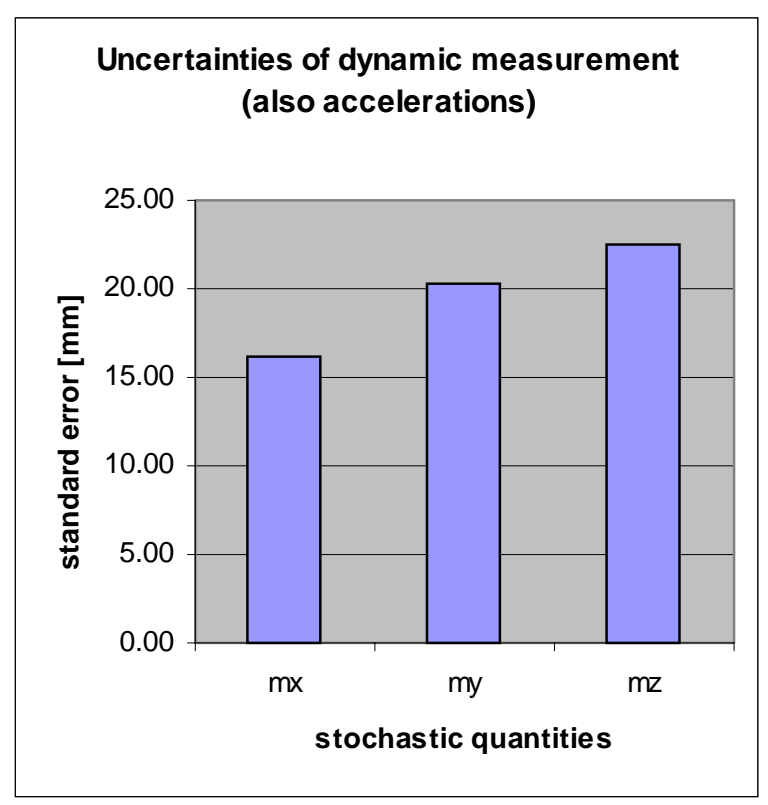

Figure 6. Random errors of dynamic measurement (static + shaking + velocities + accelerations) in the test situation.

\section{CONCLUSION}

Based on the test results, the developed stochastic models can be used to estimate in real-time the 3-D positioning accuracy of mobile work machines. The mechanisms of different random errors are complicated. The presented model is a simplified mathematical solution with which it could be possible to real-time control the behavior of random errors. In principle, it is possible to connect these models directly to a part of the automated control system of machines. It is also possible to extend these models to control the accuracy of the whole blade control system. Thus, the final accuracy of moving blades could be controlled in real-time. This technique means that more intelligence, accuracy, and reliability can be achieved in the automated control of construction. In the future, this type of intelligence could be first implemented, for example, into the automated control of a road grader. The calibration of additional uncertainties requires a series of careful tests to be generally utilized. The first calibrations have been executed in Finland during Spring-Summer 2004.

\section{REFERENCES}

Heikkilä, R. (1996) The Applicability of an Electro-Optical Coordinate Measuring Technique to the Dimensional Control of Precast Concrete Facade Panel Production. Oulu, Acta Universitatis Ouluensis, Technica C 86. Doctor's thesis. 87 p. + Appendixes. 